\title{
The effect of off-axis neutral beam injection on sawtooth stability in ASDEX Upgrade and MAST
}

I.T. Chapman, M.F. de Bock, S.D. Pinches, M.R. Turnyanskiy and the MAST Team EURATOM/UKAEA Fusion Association, Culham Science Centre, Abingdon, Oxfordshire OX14 3DB, United Kingdom

V.G. Igochine, M. Maraschek, G. Tardini and the ASDEX Upgrade Team Max-Planck Institut für Plasmaphysik, Euratom-Association, D-85748 Garching, Germany

July 28, 2009

\begin{abstract}
Sawtooth behaviour has been investigated in plasmas heated with off-axis neutral beam injection (NBI) in ASDEX Upgrade [A. Herrmann and O. Gruber, Fusion Sci. Technol. 44, 569 (2003)] and the Mega-Ampere Spherical Tokamak (MAST) [A Sykes et al., Nucl. Fusion 41, 1423 (2001)]. Provided that the fast ions are well confined, the sawtooth period is found to decrease as the neutral beam is injected further off-axis. Drift kinetic modelling of such discharges qualitatively shows that the passing fast ions born outside the $q=1$ rational surface can destabilise the $n=1$ internal kink mode, thought to be related to the sawtooth instability. This effect can be enhanced by optimising the deposition of the off-axis beam energetic particle population with respect to the mode location.
\end{abstract}




\section{INTRODUCTION}

Control of sawtooth oscillations in tokamak plasmas remains a crucial issue for present and future devices. The sawtooth instability in tokamak plasmas results in a regular periodic reorganisation of the plasma surrounding the magnetic axis. The sawtooth cycle consists of a quiescent period, during which the core plasma density and temperature increase, followed by the growth of a helical magnetic perturbation, which in turn is followed by a rapid collapse of the central density and temperature [1]. Energetic ions trapped outside the region of strongest magnetic field have been shown to stabilise the sawteeth, resulting in a longer period between relaxations $[2,3]$. This is important for ITER, where the fusion-born $3.5 \mathrm{MeV}$ alpha particles can be expected to lead to long sawtooth periods $[4,5]$. In the absence of an effective actuator to shorten the sawtooth period, coupling can occur with instabilities located at rational surfaces outside the $q=1$ surface - the location of the sawtooth instability. Furthermore, it has been shown in the Joint European Torus (JET) that long sawteeth are more likely to trigger performance-degrading neoclassical tearing modes (NTMs) at lower plasma pressures [6,7]. Conversely, improved confinement is often coincident with smaller, regular sawteeth, which have the ancillary benefit of preventing accumulation of impurities in the plasma core [8]. Consequently, many techniques have been developed to deliberately destabilise sawtooth oscillations: Electron cyclotron resonance heating has been used to control sawtooth periods, for instance in the Tokamak à Configuration Variable (TCV) [9], ASDEX Upgrade [10], JT-60U [11] and the Tokamak Experiment for Technology Oriented Research (TEXTOR) [12]. Recent results from TORE-SUPRA [13] also suggest that electron cyclotron current drive (ECCD) can be used to destabilise long-period sawteeth stabilised by a population of fast ions resulting from simultaneous ion cyclotron resonance heating (ICRH). It has also been shown that off-axis ICRH can be used to destabilise fast-ion induced long sawteeth $[6,14-16]$.

Whilst, the stabilising effects of energetic trapped particles have been appreciated for some time $[2,3]$, it has also been found that under certain conditions, untrapped - or passing - fast ions can also strongly influence sawtooth behaviour. Following results from JT-60U showing that the very large passing fraction from negative-ion neutral beam injection altered the sawtooth period significantly [17], it was suggested that sawtooth destabilisation could be achieved using NBI deeply passing ions $[18,19]$. The mechanism responsible for such sawtooth control is due to the contribution to the change in the energy of the $n / m=1 / 1$ internal kink mode resulting from passing particles that intersect the $q=1$ rational surface. This fast ion effect is enhanced when the distribution function of passing ions is distributed 
asymmetrically with respect to their velocity parallel to the magnetic field. The analytical theory has recently been extended to consider all of velocity space including barely passing energetic particles [16]. Sawtooth control due to off-axis passing ions has been demonstrated both numerically and experimentally in JET [20,21]. In reference [21] long sawtooth periods caused by on-axis heating were deliberately shortened by the additional application of off-axis NBI, despite an overall increase in the plasma pressure. In this paper we concentrate on the effects of moving the deposition location of the off-axis passing fast ions with respect to the $q=1$ surface.

The neutral beams planned for ITER will be able to tilt such that the beam deposition is moved further off-axis. In order to penetrate the hot, dense plasmas in ITER, neutral deuterium beam energies of the order of $0.5-1.0 \mathrm{MeV}$ are necessary. The beam can be aimed at two extreme (on-axis and off-axis) positions by tilting the beam source around a horizontal axis on its support flange [22]. Thus, it is of interest to consider whether tilting beams to aim further off-axis can destabilise sawtooth oscillations by the introduction of a population of energetic ions outside the $q=1$ surface. In section II we describe experiments from both ASDEX Upgrade and MAST where the NBI path has been moved further off-axis by tilting the beams and by vertically displacing the plasma, respectively. The equilibria and fast ion distribution functions from both experiments are discussed in section III, before the linear stability of the $n=1$ kink mode is assessed in section IV. Finally, the implications of these results are discussed in section V.

\section{EXPERIMENTAL RESULTS}

\section{A ASDEX Upgrade}

ASDEX Upgrade is ideally suited to investigate the potential role of off-axis NBI for sawtooth control because it has two tangential NBI sources, which inject fast ions towards the mid-radius of the plasma. Furthermore, it is also equipped with an ICRH system, which can be used to generate an energetic particle population in the core, like that expected in a burning plasma. Previous experiments in ASDEX Upgrade have considered the effect of off-axis NBI on sawtooth behaviour by moving the position of the $q=1$ surface with respect to the beam deposition location [21]. Whilst the sawtooth behaviour is clearly modified, many other plasma parameters change, most notably the toroidal field, the resonance location of the ICRH and the toroidal rotation, meaning that it is impossible to make firm inferences about the role of off-axis NBI. 
In the ASDEX Upgrade experiments reported here, the trajectory of the most off-axis positive ion neutral injector (PINI) has been changed in order to move the deposition location of the energetic ions whilst keeping the plasma conditions relatively unchanged. The sawtooth behaviour has been compared in ASDEX Upgrade plasmas with plasma current, $I_{p}=1 \mathrm{MA}$, toroidal field, $B_{T}=2.5 \mathrm{~T}$ and density $n_{e} \approx 9 \times 10^{19} \mathrm{~m}^{-3}$. In each case, a mixture of on-axis neutral beam heating and ion cyclotron resonance heating is used to establish long-period sawteeth due to the population of energetic particles in the core. Later in the discharge, the most off-axis PINI is applied, and its trajectory is varied between shots. The angle of injection is varied by setting the angle of inclination at the beam source as $\beta=6.60^{\circ}, 6.65^{\circ}, 6.68^{\circ}$ as defined in reference [23]. This corresponds to the beam trajectories shown in figure 1. Also shown for comparison is the approximate position of the $q=1$ surface as determined from the inversion radius observed in the soft X-ray emission.

In the phase when there is only on-axis heating, the sawtooth period is approximately $\tau_{s} \sim 65 \mathrm{~ms}$, though the sawteeth are interrupted by the presence of concurrent NTMs. Upon application of the offaxis NBI with the deposition oriented nearest to the magnetic axis - in discharge 24006 - the sawtooth period increases to $\tau_{s} \sim 94 \mathrm{~ms}$. As the beam is moved further off-axis the sawtooth period decreases. In shot 24005, when the beam trajectory is indicated by the solid line in figure 1 , the sawtooth period has fallen to $\tau_{s} \sim 72 \mathrm{~ms}$. Furthermore, when the beam is shifted even further off-axis and its trajectory is now indicated by the dot-dashed line in figure 1 , the period drops to $\tau_{s} \sim 64 \mathrm{~ms}$, approximately the same as in the on-axis only heating phase. The sawtooth behaviour in these three shots is illustrated in figure 2 .

In each case, the application of the tangential PINI results in an increase in the toroidal rotation of the plasma. However, there is no significant change in the amplitude of the rotation or the flow shear at $q=1$ as the beam trajectory is shifted. Thus, the sawtooth period is not strongly influenced by the rotation as the beam trajectory is moved. Whilst the change in rotation for different trajectories does not seem to be important, the increase in rotation that application of the tangential PINI causes does play a significant role in causing the increase in sawtooth period from the on-axis heating phase. Even with the most off-axis fast ion deposition, the enhanced destabilisation from the energetic particles is balanced by the increased toroidal flow, resulting in no change to the sawtooth period upon application of the off-axis beam. As discussed in reference [21], this complex competition between the destabilisation of the passing ion effects and stabilisation from increased torque means that off-axis NBI is not a practical sawtooth control actuator in ASDEX Upgrade. It should be noted that the small rotation predicted for ITER $[24,25]$ means that such a competition between kinetic and gyroscopic effects is unlikely to preclude 
off-axis NBI as a control actuator in that case.

\section{B MAST}

The effect of off-axis NBI has also been assessed in MAST. Its large vacuum vessel means that MAST is able to vary the deposition position of the NBI by moving the plasma up and down significantly. In order to move the NBI deposition location further off-axis in MAST, we have displaced the plasma vertically, such that beam deposition begins inside $q=1$ but moves to well outside the $q=1$ surface. In all the MAST plasmas reported here, the toroidal field is $B_{0}=0.4 \mathrm{~T}$ and the line-averaged density is held constant, $\bar{n}_{e} \approx 2.35 \times 10^{19} \mathrm{~m}^{-3}$. Figure 3 shows the poloidal cross-section of the MAST device, with an approximate neutral beam shadow illustrated. Also shown are the last-closed flux surface and $q=1$ surface for discharges 20092 (solid) and 20096 (dashed). When the plasma is close to double-null configuration, the fast ions born due to neutral beam injection are clearly inside the $q=1$ surface. Conversely, in shot 20096, when the plasma magnetic axis is at $Z=-18.1 \mathrm{~cm}$, the beam ion distribution function is peaked just outside the $q=1$ surface.

Figure 4 shows the sawtooth period in these MAST discharges with respect to the vertical position of the magnetic axis when the plasma current is $I_{p}=600 \mathrm{kA}$. The sawtooth period is minimised when the beam deposition is just outside the $q=1$ surface. As the beam fast ions are born further outside $q=1$, the sawtooth period begins to increase once more. Throughout the $Z$-scan the toroidal plasma rotation, and significantly the rotation at the $q=1$ surface, only alters slightly, and does not cause a substantial change in the linear kink mode stability. Further, measurements from the motional stark effect (MSE) diagnostic suggest that the neutral beam current drive (NBCD) is similar in all down-shifted plasmas, and that in any case, the change to the magnetic shear is only small due to the rather broad NBCD.

It could be speculated that the minimum in sawtooth period arises because the passing fast ion destabilisation is strongest when the gradient in the fast ion pressure is largest and that as the beams move further off-axis, $\left.\left\langle\nabla P_{h}\right\rangle\right|_{r_{1}}$ decreases, thus reducing the destabilising effect. However, as the plasma is shifted downwards the fast ion confinement is degraded, which could also result in the reduction of destabilisation from the passing fast ions. In order to distinguish between these effects, the vertical position scan was repeated at higher plasma current. Figure 5 shows the sawtooth period in MAST with respect to the vertical position of the magnetic axis when $I_{p}=800 \mathrm{kA}$. The improved confinement of the energetic particles means that the destabilising influence of the off-axis passing ions is accentuated, manifest as a continual decrease in the sawtooth period as the beam shadow is moved further off-axis. 
The sawtooth period in NBI heated plasmas is always significantly longer than Ohmic plasmas in MAST because (i) the momentum input from beam injection spins up the plasma and results in a gyroscopic stabilisation on the $n=1$ internal kink mode [26-28], and (ii) the trapped fraction is large in a spherical tokamak, and these energetic trapped particles are typically stabilising [29]. Whilst the trapped ions and rotation introduced by the NBI do influence sawtooth stability, these effects are always stabilising during the $Z$-scan, and could not explain the variation in sawtooth behaviour exhibited experimentally. The rotation profiles in discharges $20604(Z=-13.5 \mathrm{~cm}), 20603(Z=-15.8 \mathrm{~cm}), 20601(Z=-20.1 \mathrm{~cm})$ and $20610(Z=-24.5 \mathrm{~cm})$ are illustrated in figure 6 . It is evident that neither the magnitude of the toroidal velocity, nor the flow shear at $q=1$ varies significantly during this scan, and so rotation effects cannot explain the observed sawtooth behaviour. Modelling to assess the stability of the $n=1$ kink mode in these MAST experiments is presented in section IV.

\section{EQUILIBRIA AND FAST ION DISTRIBUTION FUNC- TIONS}

\section{A ASDEX Upgrade}

Equilibria have been reconstructed for discharges 24005, 24006 and 24007 at $t=3.24,3.28,3.21 \mathrm{~s}$ respectively, during the off-axis NBI heating phase and directly before a sawtooth crash in each case. The equilibria are reconstructed by supplying the pressure and current profiles together with the plasma shape to the Helena code [30]. The current profile is found from the TransP code [31]. The pressure profile is derived from diagnostic measurements; the electron temperature is found from the Thomson scattering diagnostic and the electron density is found by fitting a modified hyperbolic tangent to the the core Thomson scattering data and the Lithium beam data at the pedestal. The HELENA equilibrium is further constrained so that the $q=1$ surface is matched to the inversion radius found from the Soft X-ray diagnostic and the plasma pressure is scaled so that $\beta_{p}=p /\left(B_{a}^{2} / 2 \mu_{0}\right)$ is as calculated by TRANSP, where $B_{a}=\mu_{0} I / l$ and $l$ is the poloidal perimeter of the plasma. It is necessary to constrain the $q$-profile appropriately since the effect of the energetic passing ions is determined by the ions with orbits that cut the $q=1$ surface [18]. Although the neutral beam current drive does result in a small perturbation to the current density profile, as illustrated in figure 7 , the $q$-profile does not change significantly. Indeed, experimentally the inversion radius found from the soft X-ray emission does not change when the off-axis 
NBI is applied, indicating that the $q$-profile has not altered significantly.

In order to retain the complex dependence of the fast ion population upon pitch angle, energy and radius, the full Monte Carlo distribution function is employed in the HaGIS drift kinetic modelling. Figure 8 shows the fast ion density calculated by TRANSP averaged over all energies and pitch angles in the poloidal plane. The fast ions are peaked near the core due to the on-axis PINI. Whilst the on-axis ions dominate in this plot, it is the presence of the off-axis passing ions which strongly influences the stability of the $n=1$ internal kink mode. Although the density of these off-axis ions is not as large as the on-axis particles, the fact that their orbits intersect the $q=1$ surface and the distribution function is asymmetrically distributed with respect to the velocity parallel to the magnetic field means that they will affect the sawteeth [16]. In all the shots considered here, the energetic particles distributions are peaked around $\lambda=v_{\|} / v \sim 0.5$ and are approximately Gaussian with respect to their pitch angle at high energies. At lower energy, the beam ion population tends to isotropy. Figure 9 shows the radial dependence of the fast ion distribution. It is evident that in all cases the peak of the fast ion distribution is at a radius well outside the $q=1$ surface, such that the fast ion pressure gradient at $q=1$ is positive in all cases. Thus, analytic theory [18] predicts that the passing ions should give a destabilising contribution. This is assessed in section IV.

\section{B MAST}

Equilibria have been constructed for MAST shots $21040(Z=-13.5 \mathrm{~cm}), 20602(Z=-18.2 \mathrm{~cm})$ and 21041 $(Z=-24.5 \mathrm{~cm})$ just before the time of a sawtooth crash. The equilibria are constrained by the position of the $q=1$ surface inferred from the inversion radius found from the soft X-ray emission. The electron temperature and density profiles supplied to the HeLEnA equilibrium code [30] are measured by using the Thomson scattering diagnostic. The current density profile is found using the EFIT code [32]. Whilst the neutral beam drive current is much larger than predicted by TRANSP in ASDEX Upgrade, the change to the current profile is broad. The current density profile calculated by TRANSP is illustrated in figure 10 for MAST shot 21041, together with the neutral beam driven current. Since the beam driven current is not strongly radially localised around the $q=1$ surface, the change in the magnetic shear at $q=1$ does not vary significantly as the plasma is displaced vertically, so this cannot provide an explanation for the dramatic changes in sawtooth behaviour exhibited experimentally. The $q$-profiles obtained using the MSE diagnostic for two of these discharges are illustrated in figure 11. It should be noted that the inversion radius found from the soft X-ray emission is more core localised that the $q=1$ surface predicted by EFIT when constrained by the MSE measurement of the pitch of the magnetic field lines (the soft 
X-ray emission shows an inversion radius at $\psi_{N}=0.36$, as illustrated by the tick mark in figure 11). The MSE measures the pitch of the field lines along the path of the neutral beam, which is well away from the magnetic axis in these vertically-shifted MAST plasmas. The vertical displacement results in a greater uncertainty in the $q$-profile predicted by EFIT constrained by the MSE measurements. However, the MSE measurements can be used qualitatively to infer the changes in the magnetic shear at $q=1$. Indeed, figure 11 illustrates that $s_{1}$ only changes fractionally as the plasma is displaced from $Z=-13.5 \mathrm{~cm}(21040)$ to $Z=-24.5 \mathrm{~cm}(21041)$. The modelling presented in section IV employs equilibria derived from EFIT reconstruction constrained by the temperature and density measured by the Thomson scattering system together with the soft X-ray measurement of the inversion radius as an estimate for the position of the $q=1$ surface.

The fast ion distribution functions for these three MAST discharges have been calculated using the TransP code. The beam ion distribution function integrated over all particle energies and pitch angles is shown in the poloidal cross-section for MAST discharges 21040 and 21041 in figure 12. It is clear that in the more vertically displaced plasmas the fast ion population is further outside the plasma core. As was the case for ASDEX Upgrade, the distribution function is approximately Gaussian with respect to pitch angle, centred at $\lambda=0.5$, for high particle energies, whereas at low energy, the distribution function is more isotropic. In order to retain the complex relationship between energy, pitch angle and radius, the full Monte Carlo distribution function from TRANSP is once again employed in the HaGIS modelling. The dependence of the fast ion population upon radial location is illustrated in figure 13. It can be seen that for discharge 21040, the gradient in the fast ion pressure at $q=1$ is very small, whereas, for discharge 21041 - when the deposition is well outside the $q=1$ surface - the fast ion pressure gradient at $q=1$ is large and positive.

\section{MODELLING INTERNAL KINK MODE STABILITY}

\section{A ASDEX Upgrade}

In order to understand the sawtooth behaviour in these ASDEX Upgrade discharges, the interaction of the magnetohydrodynamic and fast particle effects must be considered, together with the changes in the magnetic shear. We present an explanation of these results by studying MHD stability in the presence of toroidal flows, and combining this with the effects of anisotropic hot ion distributions. Appropriate tools for studying these effects are the MiSHKA-F linear MHD stability code [33] and the HAGIS drift kinetic 
code [34]. MishKA-F employs the equilibrium generated by the HeLENA code [30], as discussed in section III, in order to find the growth rate of the $n=1$ internal kink mode. The static eigenfunction and the equilibrium are then supplied to HAGIS together with the fast ion distribution function from TRANSP (also detailed in section III) in order to calculate the change in the potential energy of the kink mode in the presence of the fast ions. The change in the energy of the mode, $\delta W$, is then considered in relation to the sawtooth crash trigger criteria developed in reference [4].

Porcelli et al [4] developed a model of linear stability thresholds to determine when a sawtooth crash can occur. The fundamental trigger of the sawtooth crash is the onset of an $m=n=1$ mode. The dynamics of this instability are constrained by many factors including not only the macroscopic drive from ideal MHD, but collisionless kinetic effects related to high energy particles $[18,29,35]$ and thermal particles [36], as well as non-ideal effects localised in the narrow layer around $q=1$. In heated plasmas, there are essentially two relevant sawtooth triggering criteria [4]:

$$
\begin{array}{ll}
\pi \frac{\delta \hat{W}}{s_{1}}<\hat{\rho} \text { and } s_{1}>s_{\text {crit }}(\beta) & \text { (Resistive two-fluid instability) } \\
\pi \frac{\delta \hat{W}}{s_{1}}<-\frac{\omega_{* i} \tau_{A}}{2} & \text { (Ideal instability) }
\end{array}
$$

where $\tau_{A}=\sqrt{3} R_{0} / v_{A}$, the Alfvén speed is $v_{A}=B_{0} / \sqrt{\mu_{0} M n_{0}}, \hat{\rho}=c_{\rho} \rho / \kappa_{1}^{1 / 2} r_{1}, \rho$ is the ion Larmor radius, $\omega_{* i}$ is the ion diamagnetic frequency, $\kappa_{1}$ is the elongation of the $q=1$ surface, $r_{1}$ is the radius of the $q=1$ surface, $\delta \hat{W}=c_{*} \delta W / \xi_{0}^{2} \epsilon_{1}^{2} R_{0} B^{2}, \epsilon_{1}=r_{1} / R_{0}$ is the aspect ratio at the $q=1$ surface, $\xi_{0}$ is the displacement at the magnetic axis, $c_{\rho, *}$ are constants of order unity, and $s_{1}=r /\left.q(\mathrm{~d} q / \mathrm{d} r)\right|_{q=1}$ is the magnetic shear at the $q=1$ surface. Consequently, long sawteeth can be destabilised (ie a crash can be triggered) by enhancing $s_{1}$ (through localised current drive), or through $\delta W$ reduction or reversal.

In order to model the effects of the anisotropic fast ions born due to NBI, the change in the potential energy of the $n=1$ internal kink mode caused by the fast particles is calculated [29] as

$$
\delta W_{h}=\frac{1}{2} \int \mathrm{d} \Gamma\left(m v_{\|}^{2}+\mu B\right) \delta f \sum_{m} \vec{\kappa} \cdot \vec{\xi}^{(m) *}(r, t) e^{-i(n \zeta-m \theta)}
$$

where $\theta$ is the poloidal angle, $\vec{\kappa}=\vec{b} \cdot \nabla \vec{b}$ is the magnetic curvature vector, $\vec{b}=\vec{B} / B$ and $\mathrm{d} \Gamma$ is an infinitessimal volume element of phase-space. Analytic theory [18] predicts that the effect of the passing fast ions is determined by the finite orbit width term contained within

$$
\delta W_{h}^{p} \sim \int_{0}^{r_{1}}\left(\vec{\xi} \cdot \nabla\left\langle P_{h}\right\rangle\right)(\vec{\xi} \cdot \vec{\kappa}) \mathrm{d} \vec{r}
$$

where $P_{h}$ is the hot particle pressure. As illustrated in figure 9 of reference [20], this means that onaxis co-passing and off-axis counter-passing ions will be stabilising whereas off-axis co-passing and on- 
axis counter-passing ions will be destabilising. These predictions have been experimentally verified on JET $[20,37]$ and JT-60U [17] where the toroidal flow is slow enough that the kinetic effects dominate the sawtooth behaviour.

The reconstructed equilibria for discharges 24005, 24006 and 24007 are all found to be unstable to the $n / m=1 / 1$ internal kink mode in the presence of toroidal rotation. Measurements from the charge exchange diagnostic suggest that the rotation does not change significantly as the angle of injection of the PINI is altered. MishKA-F has been used to assess the stability of the mode using the experimental flow profiles, and the change in the growth rate is insignificant as the beam is shifted further off-axis. Similarly the change in the instability drive due to the change in the magnetic shear is also assessed by MisHKA-F as the neutral beam driven current is included in the reconstructed equilibrium. The NBCD has been shown to be significant in ASDEX Upgrade plasmas under certain plasma conditions [38, 39]. However, in the plasmas reported in this work, the neutral beam driven current predicted by TRANSP leads to an insignificant perturbation to the current profile, as illustrated in figure 7 . Consequently, the change in the mode growth rate due to changes in the $q$-profile is negligible. Thus it is important to quantify the effect of the energetic particles introduced by neutral beam injection.

The NBI fast ions are represented by the distribution function of markers taken directly from the Monte-Carlo Transp code, as outlined in section III. Drift kinetic modelling using HaGIS can then be used to assess the role that both passing and trapped fast ions play in determining the mode stability. Figure 14 shows the change in the potential energy of the 1/1 kink mode due to the presence of the passing beam ions. As the peak of the deposition of the fast ion population is shifted further off-axis, and hence further from the $q=1$ surface, the destabilisation from the passing ions is enhanced. Whilst injecting the beams further off-axis results in a slightly larger trapped fraction (the trapped-passing boundary is at $\left.v_{\|} / v=\sqrt{2 r /\left[R_{0}+r\right]}\right)$, the small increase in stabilisation arising from the trapped ions is dominated by the enhanced destabilisation from the passing ions. As the passing ions are moved further off-axis the gradient of the fast ion pressure at $q=1$ increases as illustrated in figure 9 , so $\delta W_{h}^{p}$ decreases in accordance with equation 4 . This explains why moving the deposition location further off-axis results in a reduction in the sawtooth period, since $\delta \hat{W}$ in equation 1 is reduced. Figure 14 shows the real part of the mode's perturbed potential energy for different values of the peak of the deposition of the fast ions with respect to the position of the $q=1$ surface. This is done in two ways: firstly, by simulating the three ASDEX Upgrade discharges which had different deposition locations and using full TRANSP distribution functions; secondly by using a simplified representation of the fast ion population as a Gaussian with respect to pitch 
angle and slowing down with respect to energy, and then moving the peak of the distribution function radially. The model fast ion distribution function is given by

$$
f_{h}=C \exp \left[\frac{\psi-\psi_{0}}{\Delta \psi}\right] \exp \left[\frac{\lambda-\lambda_{0}}{\Delta \lambda}\right] \frac{1}{\mathcal{E}^{3 / 2}+\mathcal{E}_{c}^{3 / 2}} \operatorname{Erf}_{c}\left[\frac{\mathcal{E}-\mathcal{E}_{0}}{\Delta \mathcal{E}}\right]
$$

with $\Delta \psi=0.1, \lambda_{0}=0.5, \Delta \lambda=0.5, \mathcal{E}_{0}=80 \mathrm{keV}, \mathcal{E}_{c}=3 \mathrm{keV}$ and $\Delta \mathcal{E}=3.5 \mathrm{keV}$. The good agreement between the full distribution function results (squares) and the simplified model (line) shows that this is an acceptable approximation which gives important information about how the mode energy changes with respect to $r_{d e p}-r_{1}$. It is evident that as the fast ion population is deposited further outside the $q=1$ surface, the passing fast ions become more destabilising in these ASDEX Upgrade plasmas. The sawtooth period is also shown for comparison, and one can infer that the shorter sawtooth period results from an enhanced destabilisation from the passing fast ions. No direct comparison can be drawn between the modelling and the experimental results since the sawtooth behaviour is inherently non-linear, whereas here we consider the linear stability of the $n=1 \mathrm{kink}$ mode. In order to fully explain the sawtooth behaviour exhibited experimentally, a transport code would need to be coupled to the accurate evaluation of the $\delta W_{h}$ terms presented here and all terms evolved in time. It is, however, very plausible that the lower $\delta W_{h}$ in off-axis NBI heated plasmas does directly produce shorter sawteeth, although the modelling presented here is only qualitative with respect to comparison with experiment.

It should be noted that the destabilisation arising from the passing ions will be sensitive to the exact location of the deposition with respect to the $q=1$ rational surface. In these ASDEX Upgrade shots the destabilisation increases as the beam is tilted off-axis since the fast ion pressure gradient at $r_{1}$ increases significantly. However, the destabilisation arising from the passing ions is not necessarily maximised at the radial location at which the positive fast ion pressure gradient is maximum [21]. Since $\delta W_{h}$ is normalised by $\epsilon_{1}^{2}=r_{1}^{2} / R_{0}^{2}$, a narrower $q=1$ radius may accentuate the normalised change in the potential energy [18]. Furthermore, the finite orbit width of the passing ions means that the biggest contribution of the ions that cross the $q=1$ surface need not necessarily be at the region of the steepest gradient of the hot particle pressure.

\section{B MAST}

The numerical tools outlined in the previous section have also been applied to analyse the sawtooth behaviour exhibited in off-axis NBI experiments in MAST, details of which are given in section II. The effect of the toroidal rotation on the stability of the $n=1$ internal kink mode is assessed with the MisHKA- 
F code [33]. We find that the toroidal rotation, which is rather slow in these displaced single null discharges when compared with typical MAST plasmas, stabilises the internal kink mode, and helps to explain why the sawtooth period in all cases is over twice as long as in Ohmic plasmas. However, the toroidal rotation does not vary significantly as the plasma is moved further off-axis as illustrated in figure 6 , so this cannot explain the observed change in sawtooth behaviour. Whilst subtle variations in the flow profile can lead to substantial changes in the stability of the internal kink mode, in the MAST rotating plasmas reported here, the point of marginal stability due to rotational stabilisation is very similar. Further work to assess the sensitivity of the linear mode stability to profile variation will be reported elsewhere [40].

The influence of the energetic particles upon $n=1$ internal kink mode stability is again assessed with the drift kinetic Hagis code [34]. Figure 15 shows the contributions to the change in the potential energy of the kink mode from the trapped and passing energetic particles and the fluid drive for MAST discharges $21040(Z=-13.5 \mathrm{~cm}), 20602(Z=-18.2 \mathrm{~cm})$ and $21041(Z=-24.5 \mathrm{~cm})$, all of which had a plasma current $I_{p}=750 \mathrm{kA}$. Whilst the trapped particles are more strongly stabilising than in ASDEX Upgrade due to the enhanced trapped fraction in a spherical tokamak, the passing fast ions still play a key role in determining the sawtooth behaviour. As the plasma is moved downwards, and so the beam deposition moves further outside the $q=1$ surface, as illustrated in figure 13, the destabilisation arising due to the presence of energetic passing beam ions is enhanced. In the shots illustrated in figure 15 the plasma current is sufficiently strong that the majority of the energetic particles are well confined, and hence the passing ion effects become increasingly strong as the gradient of the fast ion pressure at $r_{1}$ increases. However, in the plasmas with $I_{p}=600 \mathrm{kA}$ detailed in figure 4 , there is a competition between destabilisation of the kink mode as the beam ions are shifted further off-axis, and enhanced prompt losses that occur with such off-axis deposition. In the most off-axis plasma with lower plasma current the loss fraction calculated by HAGIS is as high as $32 \%$, explaining why the passing ion destabilisation is diminished in this case.

It can also be seen in figure 15 that the fluid instability drive varies in these MAST discharges. As the plasma is displaced vertically in order to move the beam deposition further off-axis, the plasma shape changes slightly. In the vertical scan encompassed by the discharges illustrated in figures 4 and 5 , the average triangularity varies between $0.30<\delta_{a}<0.38$ and the elongation is in the range $1.63<\kappa_{a}<1.69$. The more off-axis plasmas tend to have a lower triangularity and elongation than plasmas near double null configuration, as can be seen in the poloidal cross-sections illustrated in figure 3. References [41-43] shows that typically smaller elongation and larger triangularity lead to increased internal kink mode stability (ie 
a smaller $\left.-\delta W_{f}\right)$. The elongation does not change significantly in these MAST plasmas, so is unlikely to change the MHD instability drive. However, the triangularity at the plasma boundary, $\delta_{a}$, changes by up to $20 \%$. Whilst this could be expected to change the mode growth rate significantly, such a change in $\delta_{a}$ results in a much smaller change in $\delta_{1}$ and $\kappa_{1}$, so the change in plasma shape only mildly affects the kink mode stability in these MAST plasmas.

Finally, the injection of off-axis neutral beams produces a significant non-inductively driven current [44]. However, whilst the beam driven current can approach nearly $30 \%$ of the inductive current, the change to the current profile is too broad to strongly affect $s_{1}$. The neutral beam driven current in the lower single-null MAST plasmas can be a significant fraction of the inductive plasma current, as shown in figure 11, though the resultant change in the magnetic shear at $q=1$ is still small, as confirmed by measurements from the MSE diagnostic. Consequently the effect on the sawtooth linear stability according to equations 1 and 2 (as the $s_{1}$ term in the denominator changes) is insignificant compared to the change in the mode potential energy due to the presence of the energetic particles.

\section{CONCLUSIONS}

It has been shown experimentally and numerically that moving the deposition of neutral beam fast ions further outside the $q=1$ surface results in an enhanced destabilisation of sawtooth oscillations. Whilst the passing ions are destabilising when injected outside the $q=1$ radius, the peak of the deposition of the beam ions must be located sufficiently far outside $q=1$ for this destabilisation to trigger sawteeth. This effect is not as localised as that from ion cyclotron resonance heating [16], but the magnitude of the destabilisation is not as large either. Counter propagating waves can be more effective than off-axis N-NBI since they are highly energetic and have strong radial shears in the parallel asymmetries of the distribution function, accentuating the destabilisation term arising from the presence of energetic passing ions. Another important consideration for the application of off-axis NBI is the role of the toroidal rotation. When the neutral beams are injected off-axis this results in an enhanced spin up of the plasma, which generally has a stabilising effect on the internal kink mode, competing with the destabilising kinetic effects. However, it should be noted that the rotation in ITER plasmas is likely to be small $[24,25]$. It has been shown in this work that the passing fast ions arising from off-axis neutral beam injection in ASDEX Upgrade and MAST play a significant role in determining sawtooth behaviour. Furthermore, the destabilisation from the passing ions has been shown to be sensitive to the location of $q=1$ with respect to the peak 
of the deposition of the fast particles. The passing ions lead to maximum destabilisation when the $q=1$ surface is well inside the beam deposition location. This means that it is unlikely that off-axis NBI could be realistically utilised as a sawtooth control mechanism since $q=1$ must be very core localised and even then, the destabilisation from kinetic effects must overcome gyroscopic stabilisation resultant from NBI torque.

\section{Acknowledgements}

The authors would like to thank Dr RJ Akers for assistance with the MAST data analysis, Dr M Wisse for analysis of MAST toroidal rotation data, Dr C Michael for assistance with current profile measurements in MAST and Dr A Stäbler for useful discussions regarding tilting the PINI in ASDEX Upgrade. This work was partly funded by the United Kingdom Engineering and Physical Sciences Research Council and by the European Communities under the contract of Association between EURATOM and UKAEA. The views and opinions expressed herein do not necessarily reflect those of the European Commission. 


\section{References}

[1] S. Von Goeler, W. Stodiek and N. Sauthoff, Phys. Rev. Lett. 331201 (1974)

[2] D.J. Campbell, D.F.H. Start, J.A. Wesson, D.V. Bartlett, V.P. Bhatnagar, M. Bures, J.G. Cordey, G.A. Cottrell, P.A. Dupperex, A.W. Edwards, C.D. Challis, C. Gormezano, C.W. Gowers, R.S. Granetz, J.H. Hammen, T. Hellsten, J. Jacquinot, E. Lazzaro, P.J. Lomas, N. Lopes Cardozo, P. Mantica, J.A. Snipes, D. Stork, P.E. Stott, P.R. Thomas, E. Thompson, K. Thomsen, and G. Tonetti, Phys. Rev. Lett., 602148 (1988)

[3] R.B. White, P.H. Rutherford, P. Colestock and M.N. Bussac, Phys. Rev. Lett., 602038 (1988)

[4] F. Porcelli, D. Boucher and M. Rosenbluth, Plasma Phys. Control. Fusion, 38, 2163 (1996)

[5] B. Hu, R. Betti and J. Manickam, Phys. Plasmas, 13, 112505 (2006)

[6] O. Sauter, E. Westerhof, M.L. Mayoral, B. Alper, P.A. Belo, R.J. Buttery, A. Gondhalekar, T. Hellsten, T.C. Hender, D.F. Howell, T. Johnson, P. Lamalle, M.J. Mantsinen, F. Milani, M.F.F. Nave, F. Nguyen, A.L. Pecquet, S. D. Pinches, S. Podda, and J. Rapp, Phys. Rev. Lett., 88, 105001 $(2002)$

[7] R.J. Buttery, T.C. Hender, D.F. Howell, R.J. La Haye, S. Parris, O. Sauter, C.G. Windsor and JET-EFDA Contributors, Nucl. Fusion, 44678 (2004)

[8] M.F.F. Nave, J. Rapp, T. Bolzonella, R. Dux, M.J. Mantsinen, R. Budny, P. Dumortier, M. von Hellermann, S. Jachmich, H.R. Koslowski, G. Maddison, A. Messiaen, P. Monier-Garbet, J. Ongena, M.E. Puiatti, J. Strachan, G. Telesca, B. Unterberg, M. Valisa, P. de Vries and contributors to the JET-EFDA Workprogramme, Nucl. Fusion, 43, 1204 (2003)

[9] C. Angioni, T. Goodman, M. Henderson and O.Sauter, Nucl. Fusion, 43, 455 (2003)

[10] A. Mück, T.P. Goodman, M. Maraschek, G. Pereverez, F. Ryter and H. Zohm, Plasma Phys. Control. Fusion, 47, 1633 (2005)

[11] Y. Ikeda, S. Ide, T. Suzuki, A. Kasugai, K. Takahashi, K. Kajiwara, A. Isayama, T. Oikawa, K. Hamamatsu, Y. Kamada, T. Fujita, K. Sakamoto, S. Moriyama, M. Seki, R. Yoshino, T. Imai, K. Ushigusa, T. Fujii, JT-60 Team, Nucl. Fusion, 42, 375 (2002) 
[12] E. Westerhof, Proc 14th Joint Workshop on Electron Cyclotron Emission and Electron Cyclotron Heating Santorini, Greece (Heliotopos Conferences Ltd) 38 (2006)

[13] M. Lennholm, L.-G. Eriksson, F Turco, F. Bouquey, C. Darbos, R. Dumont, G. Giruzzi, M. Jung, R. Lambert, R. Magne, D. Molina, P. Moreau, F. Rimini, J-L. Segui, S. Song, and E. Traisnel, Phys. Rev. Lett., 102, 115004 (2009)

[14] L.G. Eriksson, A. Mueck, O. Sauter, S. Coda, M.J. Mantsinen, M.-L. Mayoral, E. Westerhof, R.J. Buttery, D. McDonald, T. Johnson, J.-M. Noterdaeme, and P. de Vries, Phys. Rev. Lett., 92, 235004 (2004)

[15] L.G. Eriksson, T. Johnson, M.-L. Mayoral, S. Coda, O. Sauter, R.J. Buttery, D. McDonald, T. Hellsten, M.J. Mantsinen, A. Mueck, J.-M. Noterdaeme, M. Santala, E. Westerhof, P. de Vries and JET-EFDA contributors, Nucl. Fusion, 46, S951 (2006)

[16] J.P. Graves, I.T. Chapman, S. Coda, L.G. Eriksson and T. Johnson, Phys. Rev. Lett., 102, 065005 (2009)

[17] G.J. Kramer, M. Iwase, Y. Kusama, A. Morioka, M. Nemoto, T. Nishitani, K. Shinohara, S. Takeji, K. Tobita, T. Ozeki, C.Z. Cheng, G.-Y. Fu and R. Nazikian, Nucl. Fusion, 40, 1383 (2000)

[18] J.P. Graves, Phys. Rev. Lett., 92, 185003 (2004)

[19] I.T. Chapman, S.D. Pinches, J.P. Graves, R.J. Akers, L.C. Appel, R.V. Budny, S. Coda, N.J. Conway, M. de Bock, L-G. Eriksson, R.J. Hastie, T.C. Hender, G.T.A. Huysmans, T. Johnson, H.R. Koslowski, A. Krmer-Flecken, M. Lennholm, Y. Liang, S. Saarelma, S.E. Sharapov, I. Voitsekhovitch and the MAST and TEXTOR Teams and JET EFDA Contributors, Plasma Phys. Control. Fusion, 49, B385 $(2007)$

[20] I.T. Chapman, I. Jenkins, R.V. Budny, J.P. Graves, S.D. Pinches and S. Saarelma, Plasma Phys. Control. Fusion, 50, 045006 (2008)

[21] I.T. Chapman, V.G. Igochine, J.P. Graves, S.D. Pinches, A. Gude, I. Jenkins, M. Maraschek, G. Tardini, the ASDEX Upgrade Team and JET EFDA Contributors, Nucl. Fusion, 49, 035006 (2009); Proceedings 22nd Int. Conf. on Fusion Energy, Geneva, Switzerland (Vienna: IAEA) TH4-1 (2008)

[22] ITER Technical Basis for Final Design, ITER Documentation Series, \#24 (Vienna: IAEA) Chapter 2.5 , Page 2 (2001) 
[23] A. Stäbler, M. Alexander, B. Heinemann, R. Riedl, E. Speth and ASDEX Upgrade Team, in Proceedings of the 25th European Conference on Controlled Fusion and Plasma Physics, Prague (European Physical Society, Geneva) p472 (1998)

[24] M. Rosenbluth and F. Hinton, Nucl. Fusion, 3655 (1996)

[25] J.E. Rice, A. Ince-Cushman, J.S. deGrassie, L.-G. Eriksson, Y. Sakamoto, A. Scarabosio, A. Bortolon, K.H. Burrell, B.P. Duval, C. Fenzi-Bonizec, M.J. Greenwald, R.J. Groebner, G.T. Hoang, Y. Koide, E.S. Marmar, A. Pochelon and Y. Podpaly, Nucl. Fusion 471618 (2007)

[26] I.T. Chapman, T.C. Hender, S. Saarelma, S.E. Sharapov, R.J. Akers, N.J. Conway and the MAST Team, Nucl. Fusion, 461009 (2006)

[27] F.L. Waelbroeck, Phys. Plasmas, 31047 (1996)

[28] C. Wahlberg and A. Bondeson, Phys. Plasmas 7923 (2000)

[29] F. Porcelli, Plasma Phys. Control. Fusion, 33, 1601 (1991)

[30] G.T.A. Huysmans, J.P. Goedbloed and W. Kerner, Proceedings of the CP90 Conference on Computer Physics, (World Scientific, Singapore, 1991), p. 371

[31] R.V. Budny, M.G. Bell, H. Biglari, M. Bitter, C.E. Bush, C.Z. Cheng, E.D. Fredrickson, B. Grek, K.W. Hill, H. Hsuan, A.C. Janos, D.L. Jassby, D.W. Johnson, L.C. Johnson, B. LeBlanc, D.C. McCune, D.R. Mikkelsen, H.K. Park, A.T. Ramsey, S.A. Sabbagh, S.D. Scott, J.F. Schivell, J.D. Strachan, B.C. Stratton, E.J. Synakowski, G. Taylor, M.C. Zarnstorff and S.J. Zweben, Nucl. Fusion, 32429 (1992)

[32] L. Lao, J.R. Ferron, R.J. Groebner, W. Howl, H. St. John, E.J. Strait and T.S. Taylor, Nucl. Fusion, 30, $1035(1990)$

[33] I.T. Chapman, S.E. Sharapov, G.T.A. Huysmans and A.B. Mikhailovskii, Phys. Plasmas, 13062511 $(2006)$

[34] S.D. Pinches, L.C. Appel, J. Candy, S.E. Sharapov, H.L. Berk, D. Borba, B.N. Breizman, T.C. Hender, K.I. Hopcraft, G.T.A. Huysmans, W. Kerner, Comput. Phys. Commun., 111, 133 (1998) Release Version 8.08

[35] J.P. Graves, O. Sauter and N.N. Gorelenkov, Phys. Plasmas, 10, 1034 (2003) 
[36] M. Kruskal and C. Oberman, Phys. Fluids, 1, 275 (1958)

[37] I.T. Chapman, J.P. Graves, S.D. Pinches, L.C. Appel, R.J. Hastie, T.C. Hender, S. Saarelma, S.E. Sharapov, and I. Voitsekhovitch, Phys. Plasmas, 14, 070703 (2007)

[38] S. Günter, G. Conway, C. Forest, H.-U. Fahrbach, M. Garcia Munoz, S. daGraca, T. Hauff, J. Hobirk, V. Igochine, F. Jenko, K. Lackner, P. Lauber, P. McCarthy, M. Maraschek, P. Martin, E. Poli, K. Sassenberg, E. Strumberger, G. Tardini, H. Zohm and the ASDEX Upgrade Team, Proceedings 21st Int. Conf. on Fusion Energy, Chengdu, China (Vienna: IAEA) EX6-1 (2006)

[39] S. Günter, C. Angioni, M. Apostoliceanu, C. Atanasiu, M. Balden, G. Becker, W. Becker, K. Behler, K. Behringer, A. Bergmann, R. Bilato, I. Bizyukov, V. Bobkov, T. Bolzonella, D. Borba, K. Borrass, M. Brambilla, F. Braun, A. Buhler, A. Carlson, A. Chankin, J. Chen, Y. Chen, S. Cirant, G. Conway, D. Coster, T. Dannert, K. Dimova, R. Drube, R. Dux, T. Eich, K. Engelhardt, H.-U. Fahrbach, U. Fantz, L. Fattorini, M. Foley, P. Franzen, J.C. Fuchs, J. Gafert, K. Gal, G. Gantenbein, M. Garca Muoz, O. Gehre, A. Geier, L. Giannone, O. Gruber, G. Haas, D. Hartmann, B. Heger, B. Heinemann, A. Herrmann, J. Hobirk, H. Hohencker, L. Horton, M. Huart, V. Igochine, A. Jacchia, M. Jakobi, F. Jenko, A. Kallenbach, S. Klvin, O. Kardaun, M. Kaufmann, A. Keller, A. Kendl, M. Kick, J.-W. Kim, K. Kirov, S. Klose, R. Kochergov, G. Kocsis, H. Kollotzek, C. Konz, W. Kraus, K. Krieger, T. Kurki-Suonio, B. Kurzan, K. Lackner, P.T. Lang, P. Lauber, M. Laux, F. Leuterer, J. Likonen, A. Lohs, A. Lorenz, R. Lorenzini, A. Lyssoivan, C. Maggi, H. Maier, K. Mank, A. Manini, M.-E. Manso, P. Mantica, M. Maraschek, P. Martin, K.F. Mast, M. Mayer, P. McCarthy, H. Meyer, D. Meisel, H. Meister, S. Menmuir, F. Meo, P. Merkel, R. Merkel, D. Merkl, V. Mertens, F. Monaco, A. Mck, H.W. Mller, M. Mnich, H. Murmann, Y.-S. Na, R. Narayanan, G. Neu, R. Neu, J. Neuhauser, D. Nishijima, Y. Nishimura, J.-M. Noterdaeme, I. Nunes, M. Pacco-Dchs, G. Pautasso, A.G. Peeters, G. Pereverzev, S. Pinches, E. Poli, E. Posthumus-Wolfrum, T. Ptterich, R. Pugno, E. Quigley, I. Radivojevic, G. Raupp, M. Reich, R. Riedl, T. Ribeiro, V. Rohde, J. Roth, F. Ryter, S. Saarelma, W. Sandmann, J. Santos, G. Schall, H.-B. Schilling, J. Schirmer, W. Schneider, G. Schramm, J. Schweinzer, S. Schweizer, B. Scott, U. Seidel, F. Serra, C. Sihler, A. Silva, A. Sips, E. Speth, A. Stbler, K.-H. Steuer, J. Stober, B. Streibl, D. Strintzi, E. Strumberger, W. Suttrop, G. Tardini, C. Tichmann, W. Treutterer, M. Troppmann, M. Tsalas, H. Urano, P. Varela, D. Wagner, F. Wesner, E. Wrsching, M.Y. Ye, S.-W. Yoon, Q. Yu, B. Zaniol, D. Zasche, T. Zehetbauer, H.-P. Zehrfeld, M. Zilker and H. Zohm, Nucl. Fusion, 45, S98 (2005) 
[40] I.T. Chapman, J.P. Graves and C. Wahlberg, in prep Nucl. Fusion "The effect of plasma profile variation on the stability of the $n=1$ internal kink mode in rotating tokamak plasmas"

[41] J.W. Connor and R.J. Hastie, Internal Report CLM-M-106, Culham Laboratory "The effect of shaped plasma cross sections on the ideal internal kink mode in a tokamak" (1985)

[42] H. Lutjens, A. Bondeson and G. Vlad, Nucl. Fusion, 32, 1625 (1992)

[43] An Martynov, J.P. Graves and O. Sauter, Plasma Phys. Control. Fusion, 47, 1743 (2005)

[44] M.R. Turnyanskiy, D.L. Keeling, R.J. Akers, G. Cunningham, N.J. Conway, H. Meyer, C.A. Michael and S.D. Pinches, Nucl. Fusion, 49, 065002 (2009) 
Figure 1 (Color online): The beam trajectories of the off-axis PINI in ASDEX Upgrade as the PINI is tilted on its support. Also shown for comparison is the approximate position of the $q=1$ surface.

Figure 2 (Color online): The soft X-ray emission in the three ASDEX Upgrade plasmas. The sawtooth period decreases as the beam is injected further off-axis. Discharge 24006 represents the most on-axis NBI heating and shot 24007 is the most off-axis.

Figure 3 (Color online): The last-closed flux surface and the $q=1$ surface for two MAST discharges, one at $Z=-6.3 \mathrm{~cm}$ and one at $Z=-18.1 \mathrm{~cm}$, shown with respect to the approximate NBI shadow.

Figure 4 (Color online): The sawtooth period with respect to the vertical height of the magnetic axis in MAST plasmas with $I_{p}=0.6 \mathrm{MA}, B_{T}=0.4 \mathrm{~T}, n_{e}=2.35 \times 10^{19} \mathrm{~m}^{-3}$. The red and blue points correspond to the plasma cross-sections shown in figure 3 .

Figure 5 (Color online): The sawtooth period with respect to the vertical height of the magnetic axis in MAST plasmas with $I_{p}=0.8 \mathrm{MA}, B_{T}=0.4 \mathrm{~T}, n_{e}=2.35 \times 10^{19} \mathrm{~m}^{-3}$.

Figure 6 (Color online): The toroidal rotation frequency in MAST discharges $20604(Z=-13.5 \mathrm{~cm}), 20603$ $(Z=-15.8 \mathrm{~cm}), 20601(Z=-20.1 \mathrm{~cm})$ and $20610(Z=-24.5 \mathrm{~cm})$. Also shown is the position of the $q=1$ surface in each case (vertical solid lines).

Figure 7 (Color online): The current density profile for ASDEX Upgrade discharge 24007 with respect to $r / a$ as calculated by TRAnsP. The neutral beam driven current is very broad and small compared to the plasma current (here magnified by an order of magnitude for clarity). The current density profiles before the off-axis PINI is applied (2.9s) and after (3.1s) are approximately the same.

Figure 8 (Color online): The fast ion density from ASDEX Upgrade discharge 24007 at $t=3.01$ s (averaged over $0.4 \mathrm{~s}$ ) calculated by TRAnsP shown in the poloidal cross-section. The fast ions from the on-axis PINI have higher density than the off-axis ions, and so dominate this plot, even though the off-axis passing ions have a strong effect on mode stability.

Figure 9 (Color online): The fast ion distribution function with respect to $r / a$ for ASDEX Upgrade discharges 24005, 24006 and 24007, as calculated by TrANsP. The inversion radii found from the soft X-ray emission are shown for comparison.

Figure 10: The current density profile for MAST discharge 21041 as a function of $r / a$. The neutral beam driven current predicted by TRANSP is also shown, and found to be very broad and unlikely to significantly change the magnetic shear at the $q=1$ surface.

Figure 11: The $q$-profiles in MAST discharges $21040(Z=-13.5 \mathrm{~cm})$ and $21041(Z=-24.5 \mathrm{~cm})$ as measured by the MSE diagnostic. The tick mark illustrates the position of the inversion radius from the 
soft X-ray emission.

Figure 12 (Color online): Beam fast ion distribution functions at 300ms in MAST discharges 21040 $(Z=-13.5 \mathrm{~cm})$ and $21041(Z=-24.5 \mathrm{~cm})$ as calculated by TRANSP. It is clear that the more vertically displaced plasma in shot 21041 leads to a more off-axis fast ion population.

Figure 13 (Color online): The fast ion distribution function with respect to $r / a$ for MAST discharges 21040 and 21041, as calculated by TRANsP. The $q=1$ location from the sawtooth inversion radius is shown for comparison

Figure 14 (Color online): The change in the potential energy of the $n / m=1 / 1$ internal kink mode due to the presence of energetic passing ions in ASDEX Upgrade shots 24005, 24006 and 24007 as calculated by the HAGis code. As the deposition location is moved further outside $r_{1}$, the passing particles become more destabilising. This is in qualitative agreement with the sawtooth period, also shown for comparison. Figure 15 (Color online): The change in the potential energy of the $n / m=1 / 1$ internal kink mode due to the presence of energetic passing ions in MAST shots $21040(Z=-13.5 \mathrm{~cm}), 20602(Z=-18.2 \mathrm{~cm})$ and $21041(Z=-24.5 \mathrm{~cm})$ as calculated by the HAGIS code. As the deposition location is moved further outside $r_{1}$, the passing particles become more destabilising. The increased trapped fraction results in enhanced trapped ion stabilisation which is approximately balanced by increased instability drive due to reduction in elongation and triangularity. 


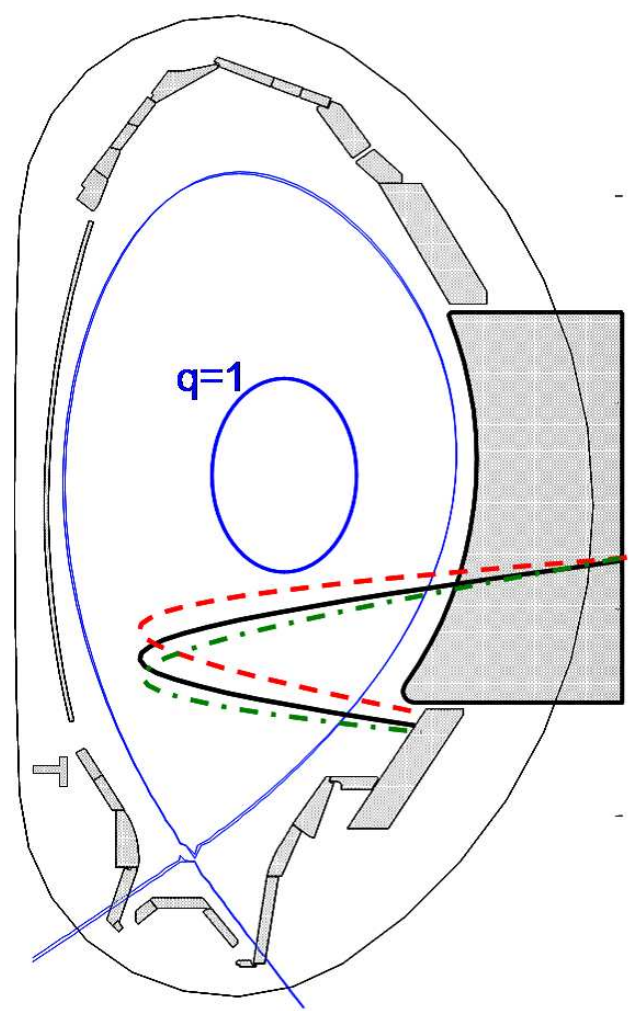

Figure 1: (Color online) The beam trajectories of the off-axis PINI in ASDEX Upgrade as the PINI is tilted on its support. Also shown for comparison is the approximate position of the $q=1$ surface.

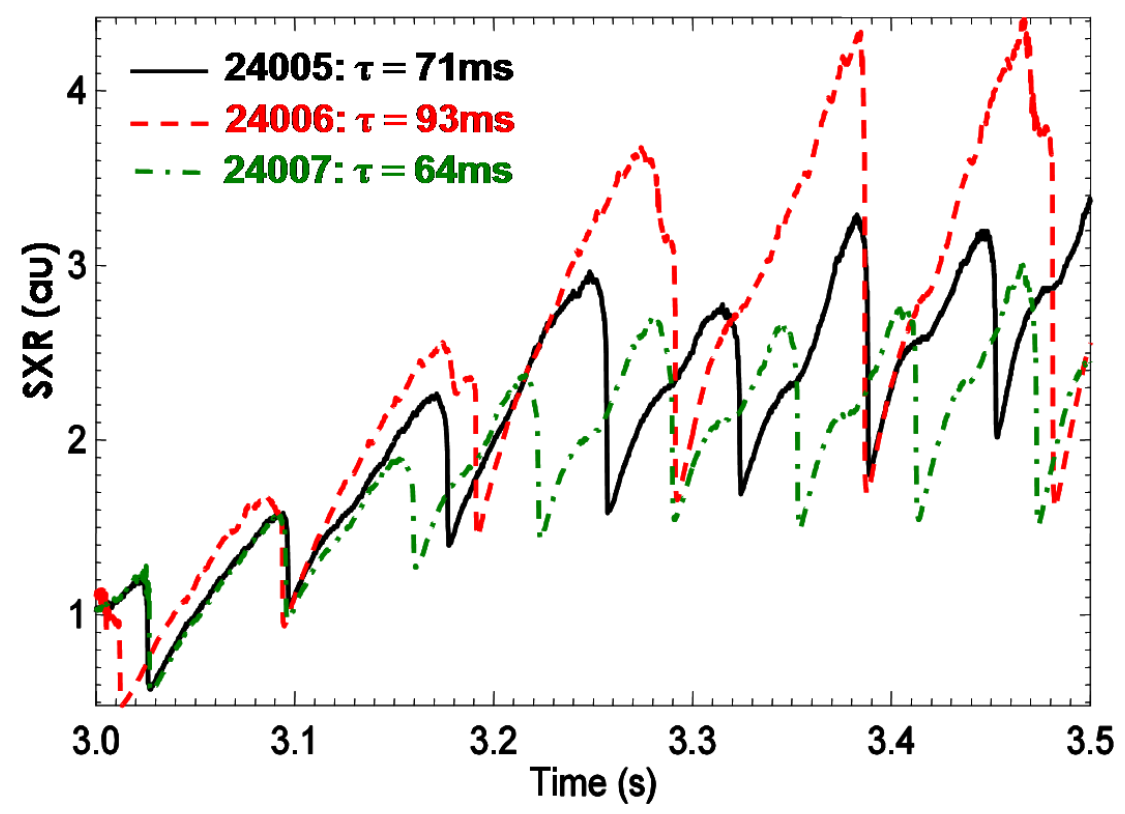

Figure 2: (Color online) The soft X-ray emission in three ASDEX Upgrade plasmas. The sawtooth period decreases as the beam is injected further off-axis. Discharge 24006 represents the most on-axis NBI heating and shot 24007 is the most off-axis. 


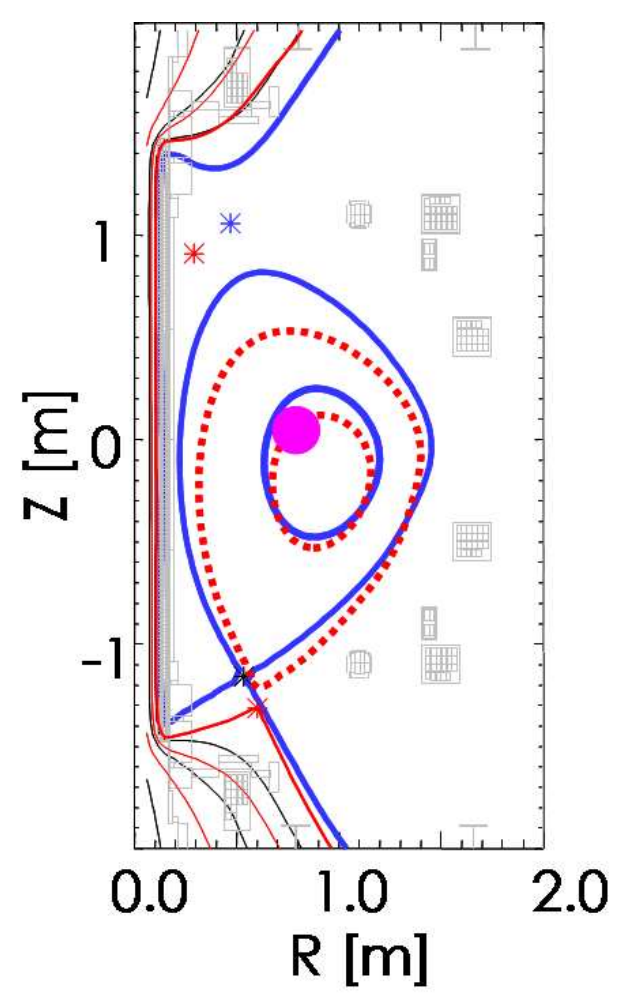

Figure 3: (Color online) The last-closed flux surface and the $q=1$ surface for two MAST discharges, one at $Z=-6.3 \mathrm{~cm}$ and one at $Z=-18.1 \mathrm{~cm}$, shown with respect to the approximate NBI shadow.

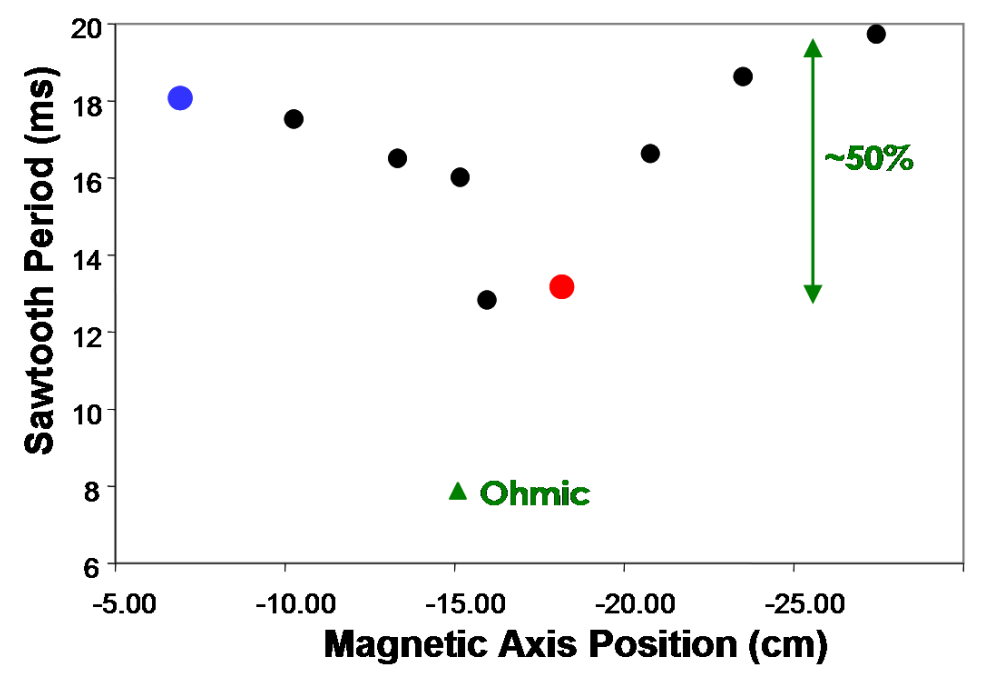

Figure 4: (Color online) The sawtooth period with respect to the vertical height of the magnetic axis in MAST plasmas with $I_{p}=0.6 \mathrm{MA}, B_{T}=0.4 \mathrm{~T}, n_{e}=2.35 \times 10^{19} \mathrm{~m}^{-3}$. The red and blue points correspond to the plasma cross-sections shown in figure 3. 


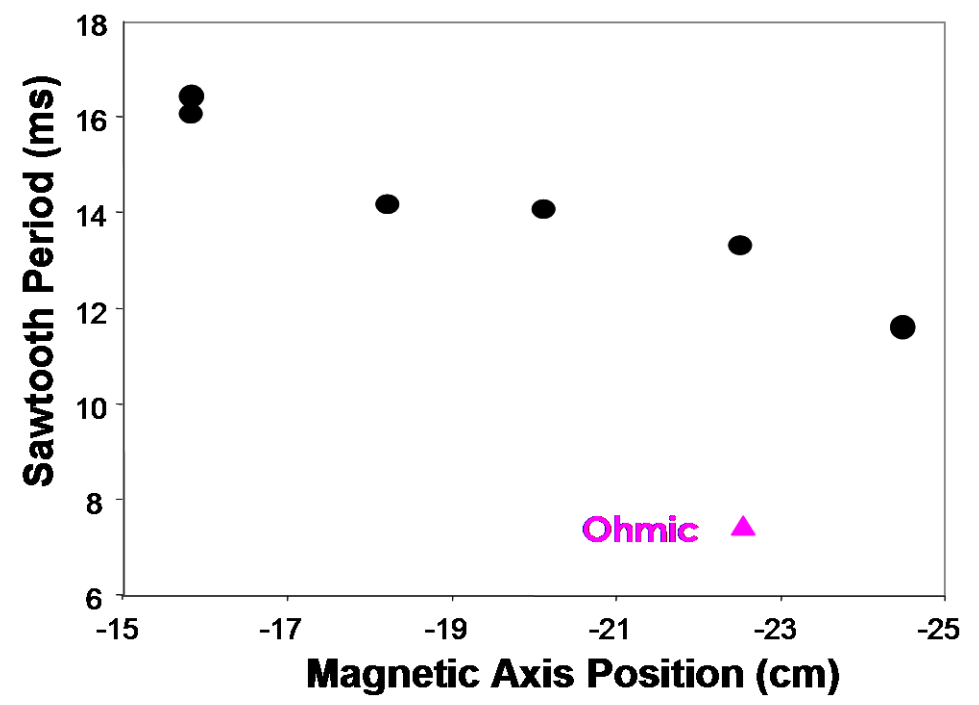

Figure 5: (Color online) The sawtooth period with respect to the vertical height of the magnetic axis in MAST plasmas with $I_{p}=0.8 \mathrm{MA}, B_{T}=0.4 \mathrm{~T}, n_{e}=2.35 \times 10^{19} \mathrm{~m}^{-3}$.

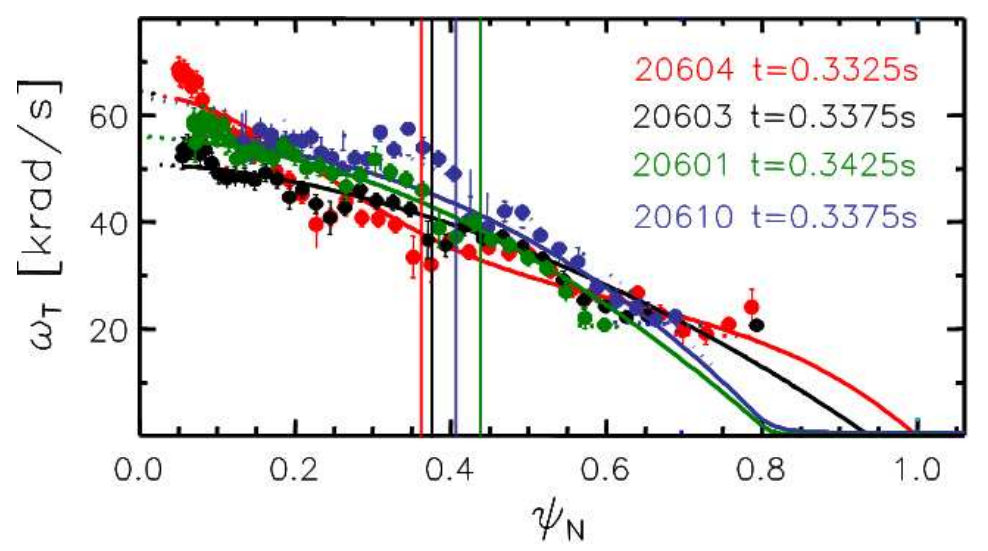

Figure 6: (Color online) The toroidal rotation frequency in MAST discharges $20604(Z=-13.5 \mathrm{~cm}), 20603$ $(Z=-15.8 \mathrm{~cm}), 20601(Z=-20.1 \mathrm{~cm})$ and $20610(Z=-24.5 \mathrm{~cm})$. Also shown is the position of the $q=1$ surface in each case (vertical solid lines). 


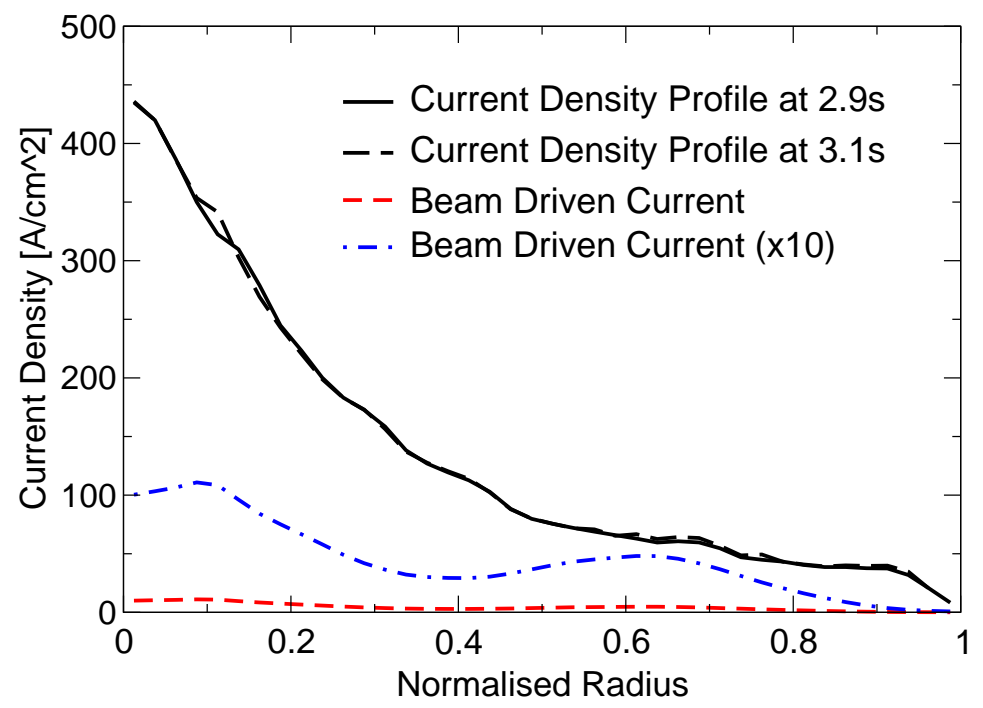

Figure 7: (Color online) The current density profile for ASDEX Upgrade discharge 24007 with respect to $r / a$ as calculated by TrANSP. The neutral beam driven current is very broad and small compared to the plasma current (here magnified by an order of magnitude for clarity). The current density profiles before the off-axis PINI is applied (2.9s) and after (3.1s) are approximately the same. 


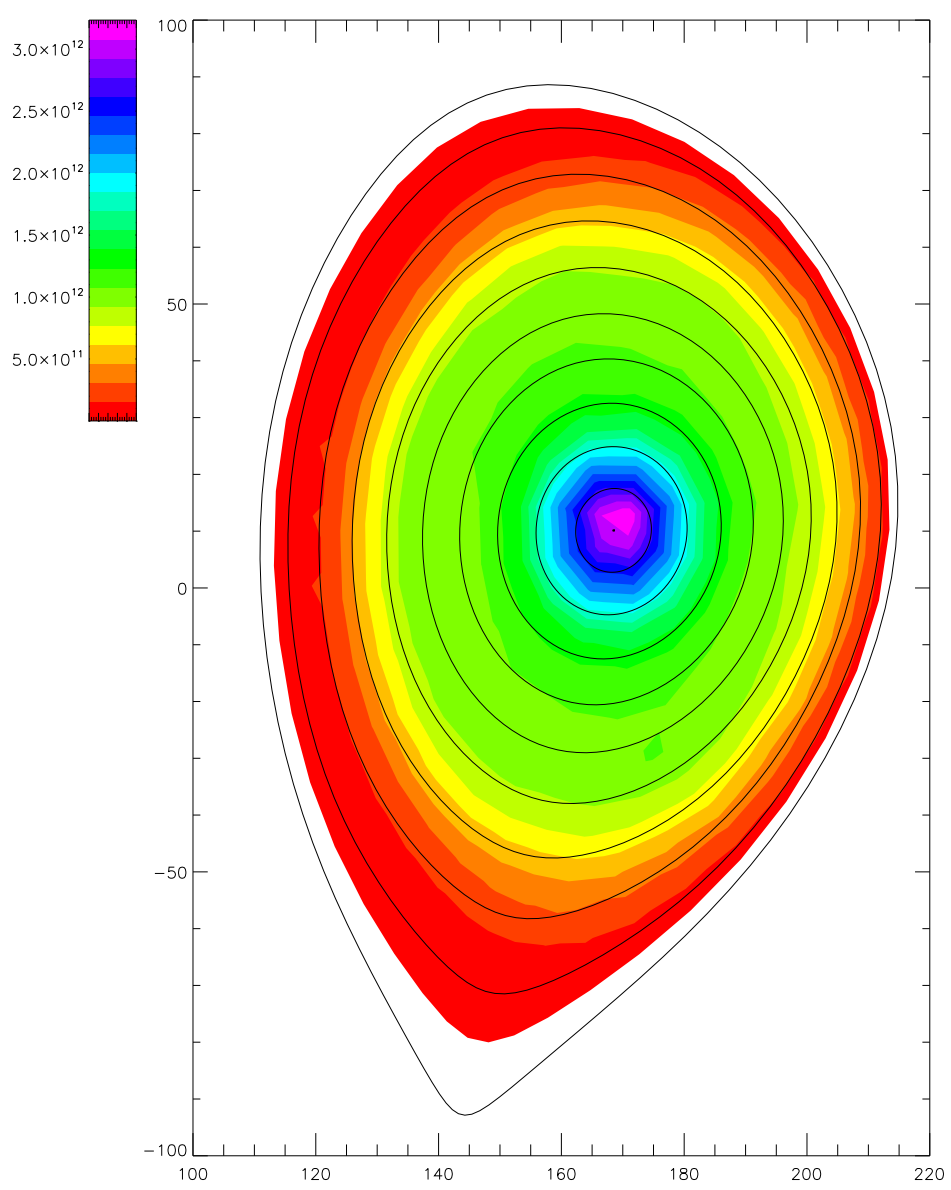

Figure 8: (Color online) The fast ion density from ASDEX Upgrade discharge 24007 at $t=3.01 \mathrm{~s}$ (averaged over $0.4 \mathrm{~s}$ ) calculated by TRANSP shown in the poloidal cross-section. The fast ions from the on-axis PINI have higher density than the off-axis ions, and so dominate this plot, even though the off-axis passing ions have a strong effect on mode stability. 


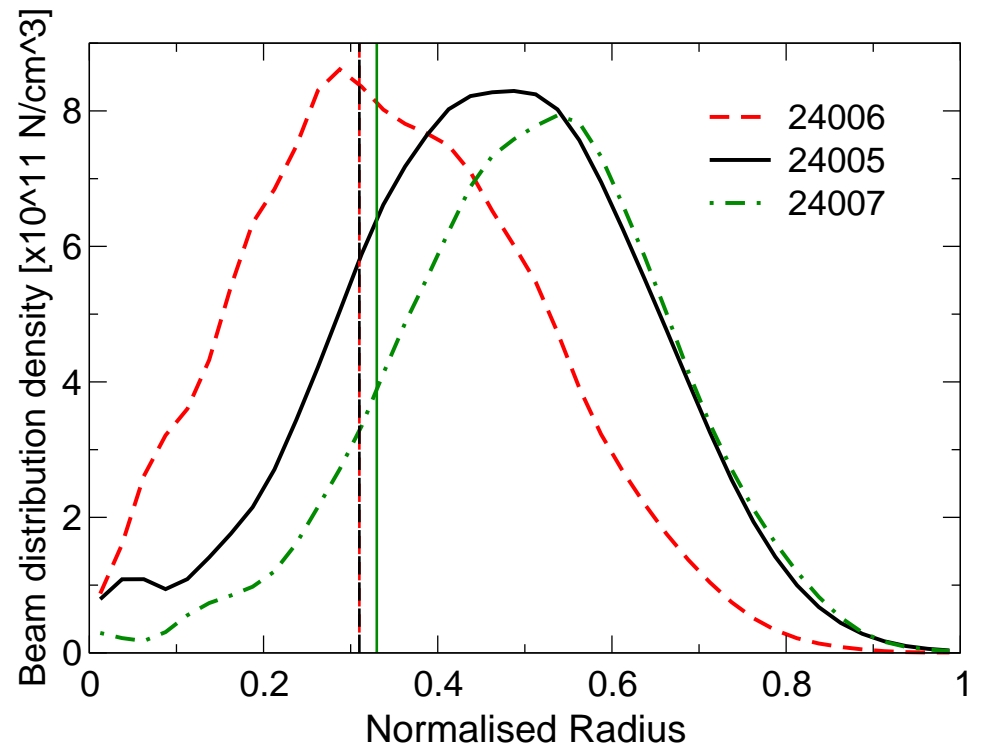

Figure 9: (Color online) The fast ion distribution function with respect to $r / a$ for ASDEX Upgrade discharges 24005, 24006 and 24007, as calculated by TrANsP. The inversion radii found from the soft $\mathrm{X}$-ray emission are shown for comparison.

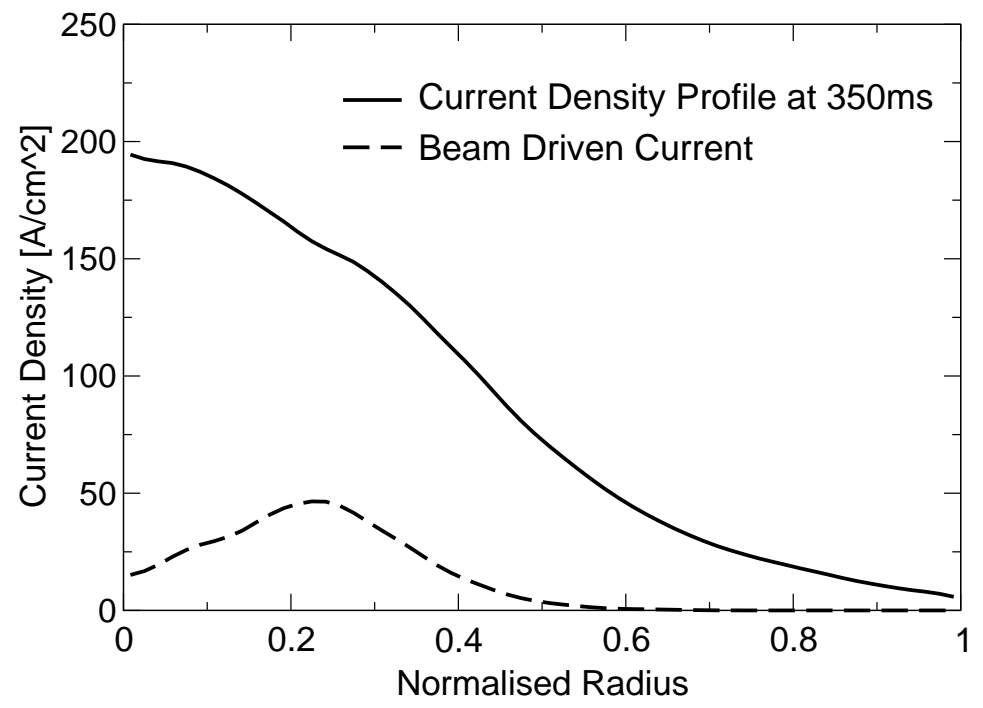

Figure 10: The current density profile for MAST discharge 21041 as a function of $r / a$. The neutral beam driven current predicted by TRANSP is also shown, and found to be very broad and unlikely to significantly change the magnetic shear at the $q=1$ surface. 


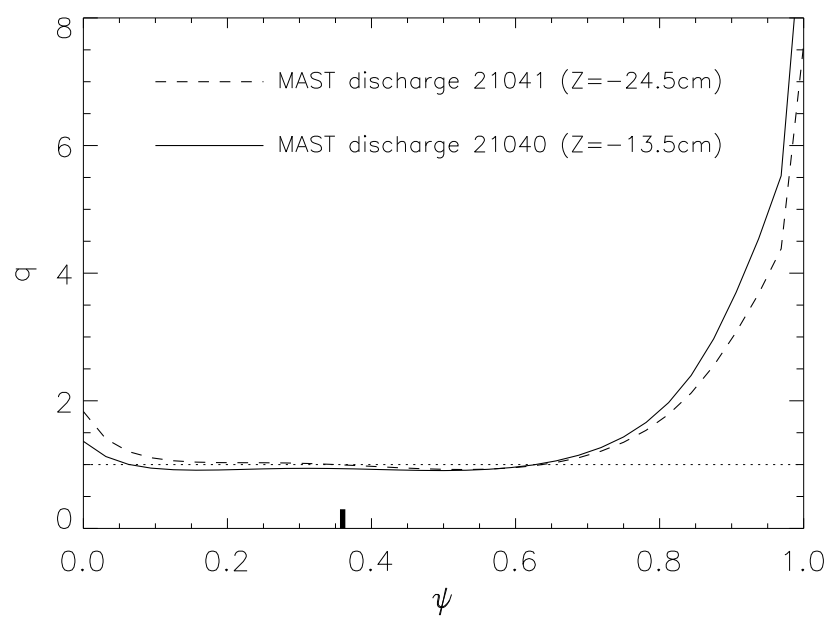

Figure 11: The $q$-profiles in MAST discharges $21040(Z=-13.5 \mathrm{~cm})$ and $21041(Z=-24.5 \mathrm{~cm})$ as measured by the MSE diagnostic. The tick mark illustrates the position of the inversion radius from the soft X-ray emission.
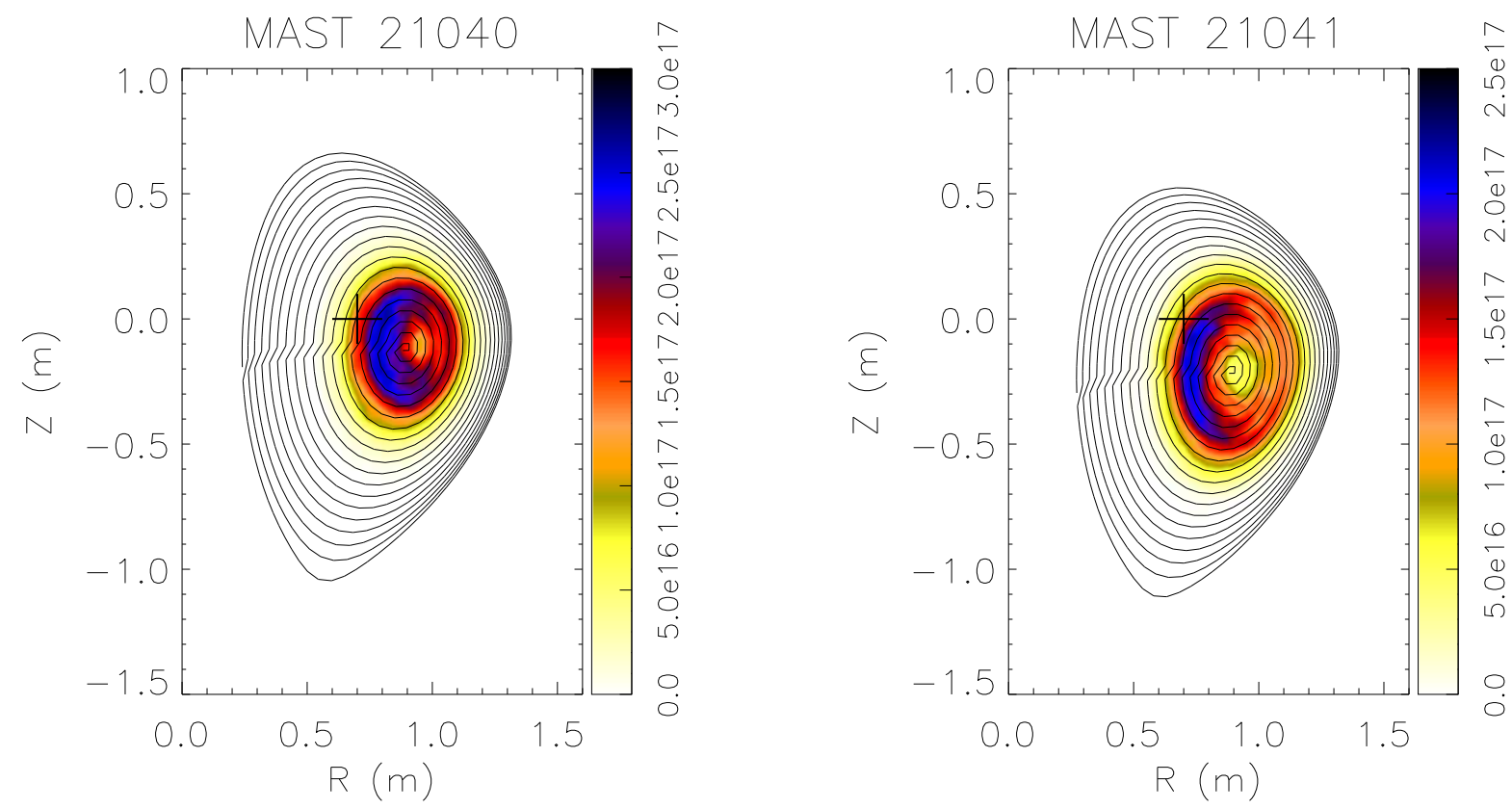

Figure 12: (Color online) Beam fast ion distribution functions at 300ms in MAST discharges 21040 $(Z=-13.5 \mathrm{~cm})$ and $21041(Z=-24.5 \mathrm{~cm})$ as calculated by TRANSP. It is clear that the more vertically displaced plasma in shot 21041 leads to a more off-axis fast ion population. 


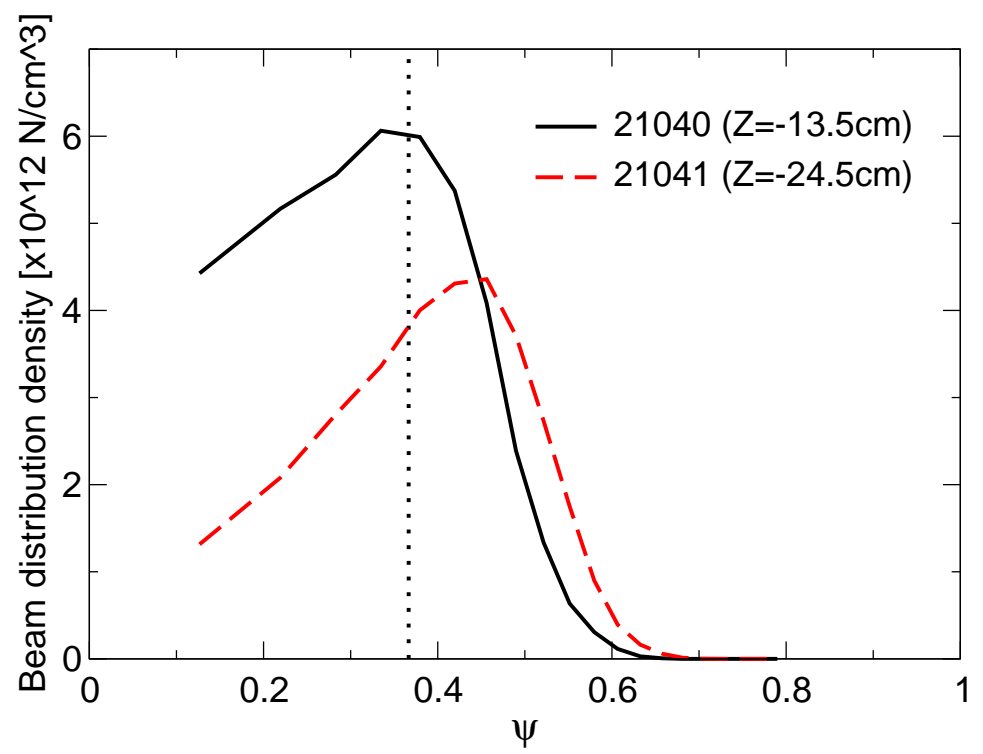

Figure 13: (Color online) The fast ion distribution function with respect to $\psi_{N}$ for MAST discharges 21040 and 21041, as calculated by TRAnsP. The $q=1$ location from the sawtooth inversion radius is shown for comparison.

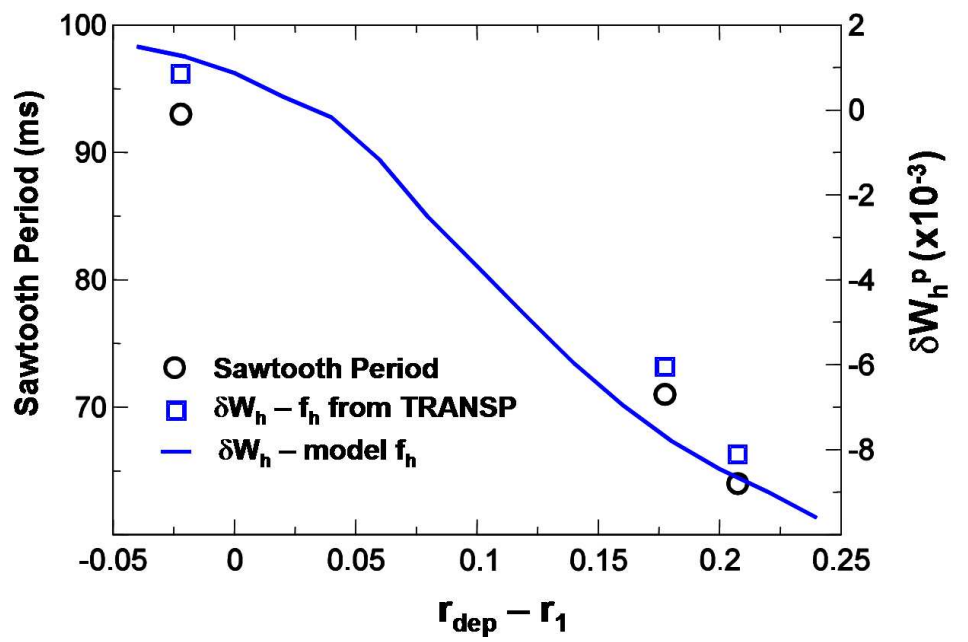

Figure 14: (Color online) The change in the potential energy of the $n / m=1 / 1$ internal kink mode due to the presence of energetic passing ions in ASDEX Upgrade shots 24005, 24006 and 24007 as calculated by the HAGis code. As the deposition location is moved further outside $r_{1}$, the passing particles become more destabilising. This is in qualitative agreement with the sawtooth period, also shown for comparison. 


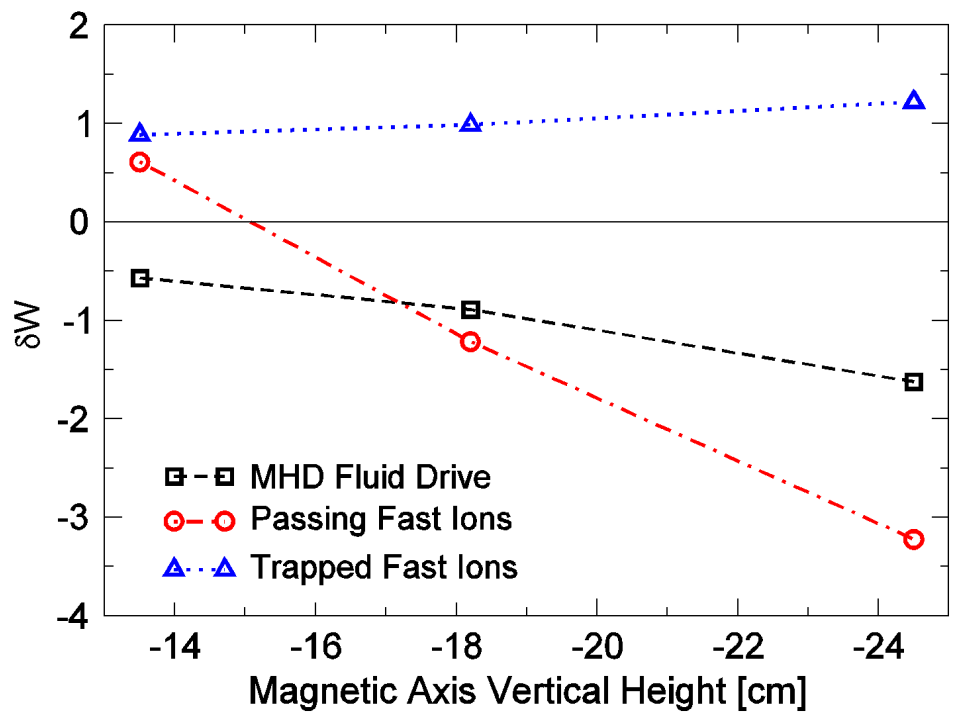

Figure 15: (Color online) The change in the potential energy of the $n / m=1 / 1$ internal kink mode due to the presence of energetic passing ions in MAST shots $21040(Z=-13.5 \mathrm{~cm}), 20602(Z=-18.2 \mathrm{~cm})$ and $21041(Z=-24.5 \mathrm{~cm})$ as calculated by the HAGIS code. As the deposition location is moved further outside $r_{1}$, the passing particles become more destabilising. The increased trapped fraction results in enhanced trapped ion stabilisation which is approximately balanced by increased instability drive due to reduction in elongation and triangularity. 